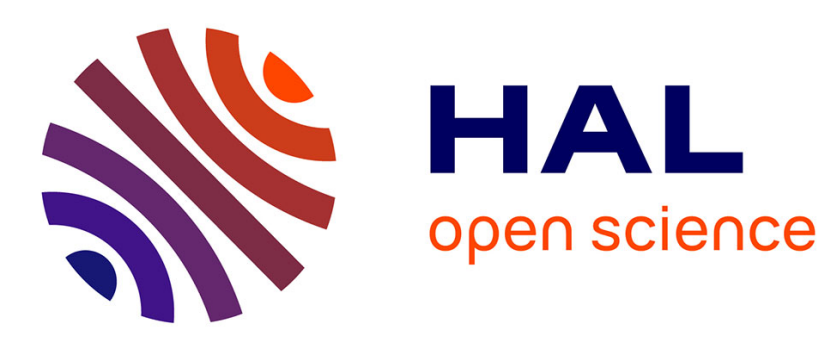

\title{
Modeling and Nonlinear Adaptive Control for Autonomous Vehicle Overtaking
}

Plamen Petrov, Fawzi Nashashibi

\section{To cite this version:}

Plamen Petrov, Fawzi Nashashibi. Modeling and Nonlinear Adaptive Control for Autonomous Vehicle Overtaking. IEEE Transactions on Intelligent Transportation Systems, 2014, 15 (4), pp.14. 10.1109/TITS.2014.2303995 . hal-01107533

\section{HAL Id: hal-01107533 \\ https://inria.hal.science/hal-01107533}

Submitted on 20 Jan 2015

HAL is a multi-disciplinary open access archive for the deposit and dissemination of scientific research documents, whether they are published or not. The documents may come from teaching and research institutions in France or abroad, or from public or private research centers.
L'archive ouverte pluridisciplinaire HAL, est destinée au dépôt et à la diffusion de documents scientifiques de niveau recherche, publiés ou non, émanant des établissements d'enseignement et de recherche français ou étrangers, des laboratoires publics ou privés. 


\title{
Modeling and Nonlinear Adaptive Control for Autonomous Vehicle Overtaking
}

\author{
Plamen Petrov and Fawzi Nashashibi
}

\begin{abstract}
In this paper, we present a nonlinear adaptive controller for a two-vehicle automated overtaking maneuver. We consider the problem of an autonomous three-phase overtaking without the use of any roadway marking scheme or inter-vehicle communication. The developed feedback controller requires information for the current relative intervehicle position and orientation available from onboard sensors only. We apply standard robotic nomenclature for translational and rotational displacements and velocities and propose a general kinematic model of the vehicles during the overtaking maneuver including for the relative inter-vehicle kinematics. The overtaking maneuver is investigated as a tracking problem with respect to desired polynomial virtual trajectories for every phase, which are generated in real time. An update control law for the automated overtaking vehicle is designed that allows tracking the desired trajectories in the presence of unknown velocity of the overtaken vehicle. Simulation results illustrate the performance of the proposed controller.
\end{abstract}

\section{INTRODUCTION}

$\mathrm{I}_{\mathrm{r}}^{\mathrm{N}}$ $\mathrm{N}$ recent years, there have been significant interest and research activity in the area of cooperative control of multiple automated vehicles. The ultimate goal in automating the driving process is to reduce accidents caused by human error and improve safety. In the same time, the full automation can greatly increase the roadway capacity and diminish air pollution by efficient use of fuel. The movement of the automated vehicles is realized through different maneuvers like lane following, lane change, merging, splitting, platooning, and overtaking. Fully automated vehicle operation has been investigated, prototyped and demonstrated in several projects during the last decades for different kinds of maneuvers. One of the earliest experiments was conducted in the framework of the French project ARAMIS [1] with automatic platooning, insertions and exits. During the 1990s, vehicle platooning was investigated in several programs in Europe, US and Japan. Within the PRAXITELE project [2], in 1994, INRIA demonstrated a vision-based control coupling of car-sharing electric vehicles with fully automatic driving capability so that they could automatically link up in a platoon and be redistributed across parking areas. In 1997, California's PATH (Partners for Advanced Transit Highways) team conducted a demonstration of close-headway platooning under fully automated control [3]. At DEMO'97, the eight PATH

The authors acknowledge the financial support from the FP7 EU project HAVEit.

P. Petrov is with the Faculty of Mechanical Engineering, Technical University of Sofia, 1000 Sofia, Bulgaria, (phone: 3592-965-3271; email: ppetrov@tu-sofia.bg).

F. Nashashibi is with the Institut National de la Recherche en Informatique et Automatique (INRIA), 78153 Rocquencourt, France, (email: Fawzi.Nashashibi@inria.fr). vehicle platoon demonstrated functions including lane keeping, lane change, platoon split and merge maneuvers. The system used inter-vehicle communications providing vehicle speed and acceleration combined with radar information to achieve tightly coordinate maneuvering. The roadway was instrumented with magnetic markers placed along the centerlane, which enabled the vehicle to measure its lateral position relative to the lane center. More recently, the IMTS (Intelligent Multimode Transit System) developed by Toyota and implemented as a parking shuttle at the 2005 World Exposition in Aichi, Japan, provided a new public transport system of automated buses running in platoons on dedicated lines using magnetic markers and high-frequency radars. [4]. In Europe, a series of EU-funded projects: Cybercars and CyberCars 2 [5], STARDUST [6], CyberMove [7], CityMobil [8], HAVEit [9] were addressed the issue of automated driving like adaptive cruise control, lane keeping, lane change, stop\&go and platooning. The main objective of these projects was to accelerate the development and implementation of cybernetic transportation systems for people and goods in urban environments.

The automated vehicle overtaking is one of the most complex maneuvers for road automation. In contrast to lane keeping and lane change maneuvers, the overtaking is a composition of three consecutive maneuvers: lane change followed by traveling a specified path parallel to the overtaken vehicle in an adjacent line (lane keeping), and again a lane change, which have to be planned and coordinated. Lane keeping is a fundamental design challenge and a long-term field of extensive research and development of automated vehicles. The lane keeping system deals with the automatically controlling the steering to keep the automated vehicle to travel along a prescribed path. Often the automatic control of the longitudinal and lateral motion of the vehicle is separately undertaken, and each controller is designed as if the longitudinal and steering vehicle dynamics is decoupled [10]. Different lateral controllers have been proposed based on either linearized vehicle models as the "bicycle model" [11], or nonlinear vehicle models [11] in terms of longitudinal vehicle velocity and wheel sideslip angles. For low speed motion (for speeds less than $5 \mathrm{~m} / \mathrm{s}$, [13]), which is often the case of automated vehicles, a reasonable assumption is that the slip angles of the wheels are zero. In this case, the velocity vectors of the wheels are in the direction of the orientation of the wheels and classical methods from analytic mechanics can be used to develop mathematical models of the vehicle as a nonholonomic system [14, 15]. A linear time-invariant lane keeping controller, based on the bicycle model was presented in [16], where the vehicle tracks a desired path with constant velocity. Since the 
vehicle model depends on the vehicle speed, it was pointed out, that with increasing the longitudinal vehicle velocity, the poles of the closed-loop system move towards the imaginary axis, which reduces the stability of the system. An adaptive lane keeping controller was proposed in [17] to overcome this problem. In order to deal with the vehicle dynamics uncertainty and parameter variation, robust $\left(\mathrm{H}_{\infty}\right.$ [18], sliding mode [19]) and adaptive [20] lane keeping controllers were proposed. The coupling between the longitudinal and lateral dynamics can become appreciable, for example, during sever road conditions or inclement weather. A combined longitudinal and lateral controller which achieves asymptotically stable ideal trajectory without measurement of the lateral vehicle velocity and accurate knowledge of the vehicle parameters was reported in [21]. Lane keeping systems are also under development by several automotive manufacturers [22]. The objective of the lane change maneuver is to transfer a vehicle which is under lane following control to lane following in an adjacent lane [23]. During the lane change maneuver, the vehicle should have predefine (desired) trajectory to track which needs to comply with several feasibility requirements as maximum lateral acceleration and maximum steering angle, arising from the characteristics of the vehicle [24]. The existing desired trajectories can be categorized based on the types of curves that generate: circular [25], harmonic [26], spline [27], polynomial [28 ,29, 30] line segments, which, in general, should be functions of time. Nonlinear control techniques as sliding mode [31, 32] and robust switching control [33] have been used for designing lane change controllers.

Compared to lane keeping and lane changing, overtaking is even more challenging problem, because it is a composition of consecutive maneuvers, which have to be coordinated. During the overtaking maneuver, the overtaking vehicle is transferred temporally from one lane in an adjacent lane (lane change), followed by lane tracking and again - returning in the same lane. In the automated vehicle overtaking, the control objective can be realized using infrastructure-supported approach or in autonomous fashion. The infrastructure-supported approach is based on trajectories, which can be physically or virtually marked, in combination with inter-vehicle communication. In this case, the system is not completely interconnected, since during the second phase (lane keeping) of the overtaking maneuver, each vehicle independently follows proper reference lane. In the autonomous overtaking approach, only on-board sensors are used to determine the relative position and orientation between vehicles without any roadway marking scheme or inter-vehicle communication. The steering commands for the controlled vehicle are set according to the relative position and orientation with respect to the overtaken car and, in that way, the overtaking vehicle accomplishes the maneuver with respect to the overtaken vehicle instead of the road [34]. An autonomous overtaking control system may constitute a backup system for the infrastructurebased control system when the letter is malfunctioning or due to the failure the inter-vehicle communication. While considerable research work has been reported on lane keeping and lane change maneuver, the problem of automated overtaking has attracted less attention. In [35], an optimal trajectory for an overtaking maneuver was designed by formulating a nonlinear constrained optimization problem. The solution of the optimization problem determines the optimal time and distance for the lane change maneuver using 5-th degree polynomial functions. An on-line time-optimal trajectory planning algorithm for the guidance of a pursuer vehicle overtaking a slower vehicle which is based on Rendezvous-Guidance principle was proposed in [36]. Two-layer controller architecture for overtaking maneuver is presented in [37]. The lower level consists of two fuzzy steering controllers for path tracking and lane change, and the high-level is to evaluate the necessity and possibility of overtaking, and to switch between the low-level controllers. The route tracking system is based on the information supplied by the GPS, which digitally maps the driving zone for circulation and the vehicles know theirs positions on the road using wireless communication, (in the framework of Autopia program).

An overtaking control method based on the estimation of the conflict probability as safe indicator was proposed in [38]. The proposed method uses model predictive control and integrates decision making and control of the overtaking maneuver into a tracking control problem. An on-road demonstration of cooperative driving solutions and, in particular, overtaking maneuver by autonomous road vehicles designed for the cities is reported in [39].

This paper presents a nonlinear adaptive controller for a two-vehicle automated overtaking maneuver. We consider the problem of autonomous overtaking without the use of road infrastructure with only the current inter-vehicle position and orientation available for feedback control. Our approach consists in consecutive tracking of reference virtual points, which are positioned at desired a priory known distances from the overtaken vehicle with a virtual reference point attached to the overtaking vehicle. The reference trajectories for the desired motion of the overtaking vehicle during the overtaking maneuver are generated using polynomial functions. The overtaking maneuver is considered as a tracking problem. An adaptive nonlinear controller for the overtaking vehicle is designed that allows tracking desired trajectories in the presence of unknown velocity of the overtaken vehicle.

The organization of the paper is as follows: In Section II, the mathematical description of a three-phase overtaking maneuver suitable for feedback control is derived. The Problem formulation is given in Section III. In Section IV, a nonlinear adaptive control law is designed. Section V contains simulation results. Conclusions are presented in Section VI.

\section{MATHEMATIC MODEL}

\section{A. Three-Phase Overtaking Maneuver}

In this paper, the overtaking involving two vehicles is established as a three-phase maneuver, as illustrated in Fig. 1. 


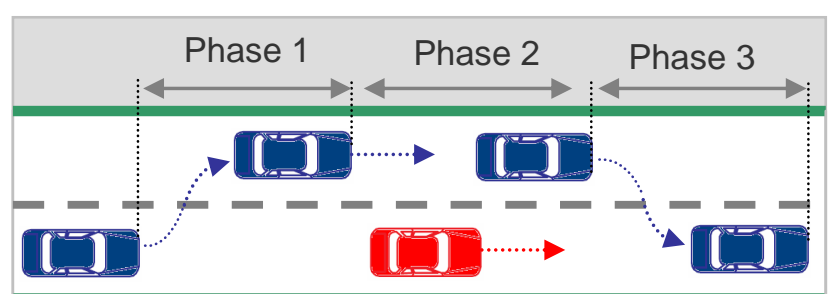

Fig. 1. A three-phase overtaking maneuver involving two vehicles

During the overtaking maneuver, we assume that the following conditions are met:

--The overtaken vehicle is moving along a rectilinear route with a constant velocity.

--The linear velocity of the overtaken vehicle is unknown.

--The available information for feedback control is the relative inter-vehicle position and orientation.

A brief description of the three-phase overtaking scenario considered in this paper is provided below. Assume that the automated vehicle has to pass the preceding car, which is at distance $d$ ahead. The first phase consists of "lane-change"-type maneuver. Starting with some initial conditions, the overtaking vehicle diverts from the lane and tracks a given reference trajectory for a given time period and has to reach a preselected position behind the overtaken vehicle, (Fig. 1). At the end of the first time period, the guidance program switches automatically from Phase 1 to Phase 2. The second phase consists of driving straight on along the overtaken vehicle at a prescribed lateral distance. The vehicle tracks again a given reference trajectory for a given time period until the overtaken vehicle has been passed and the overtaking vehicle reaches a pre-selected position on the left side of the overtaken vehicle, (Fig. 1). During the third phase, given a reference trajectory for the third phase, the overtaking vehicle returns to the lane and has to reach a pre-selected position in front of the overtaken vehicle. The desired position and linear velocity of the overtaking vehicle with respect to the overtaken vehicle at the end of every phase, as well the phase duration, are determined to satisfy the operational requirements imposed on such a maneuver. A reference trajectory for the overtaking vehicle is generated in real time for every phase. A specific feature of the proposed trajectory planning procedure is that at the beginning of each phase, the desired initial position and velocity of the overtaking vehicle coincides with its current position and velocity. In such way, there is no restriction for the initial position, orientation, linear and angular velocities of the overtaking vehicle in the beginning of every phase of the overtaking maneuver. Furthermore, smooth transition between the adjacent phases is assured. However, from a practical point of view, a minimal inter-vehicle distance $d$ is introduced for the beginning of the maneuver, in order to meet some geometrical and dynamical limitations of the vehicles, (for example, limitations for the front-wheel steering angle).

\section{B. Vehicle Kinematics}

\section{1) Coordinate System Assignments}

In this section, we apply standard robotic nomenclature for translational and rotational displacements and velocities, and methodology [40] to model the kinematics of the vehicle during the overtaking maneuver. Fig. 2 depicts the schematic of the vehicles considered in this paper. From now on, the index $i=1$ and $i=2$ correspond to the overtaken vehicle, overtaking vehicle, respectively. The vehicles have four non-deformable wheels. The wheels are assumed to roll on a horizontal plane without slipping. The longitudinal base $P_{i} S_{i}$ of the vehicle is denoted by $l_{i}$, $(i=1,2)$. To simplify the derivation of the vehicle control algorithms, we use a planar bicycle $2 \mathrm{DOFs}$ vehicle model where two virtual wheels are located at the midpoints of the front and rear wheel axles, (Fig. 2). Although these two wheels do not exist, it is assumed that they comply with the wheel rolling without slipping conditions. In order to describe the position and orientation of the vehicles in the plane during the overtaking maneuver, we assign the following coordinate frames (Fig. 2).

- Fxy - inertial coordinate frame in the plane of motion;

- $\quad P_{i} x_{P i} y_{P i},(i=1,2)$ - vehicle coordinate frame located at the center of the rear vehicle axis, where $x_{i}$ is along the longitudinal base of the vehicle. The coordinates of a reference point $P_{i}$ placed at the center of the rear vehicle axle, with respect to Fxy, are denoted by $\left(x_{P i}, y_{P i}\right)$.

- $\quad R_{j} x_{R j} y_{R j},(j=1,2,3)$ - virtual reference frames rigidly attached to the overtaken vehicle and located at prescribed places, as shown in Fig. 2. The coordinates of the origins $R_{j}$ with respect to $P_{I} x_{P I} y_{P I}$ are $\left(L_{t j}, L_{n j}\right), \quad(j=1,2)$ and the corresponding axes are parallel to those of $P_{I} x_{P I} y_{P I}$

- $L x_{L} y_{L}$ - coordinate frame rigidly linked to the overtaking vehicle and located at the mid-point of the front bumper of the vehicle at a distance $L_{2}$ from point $P_{2}$, where the $x_{L}$ axis is in direction of the longitudinal vehicle axle.

- $S_{i} x_{S i} y_{S i},(i=1,2)$ - wheel coordinate system with origin placed at the coordinate center of the virtual front steering wheel; the $x_{s i}$ axis is in the direction of the wheel orientation.

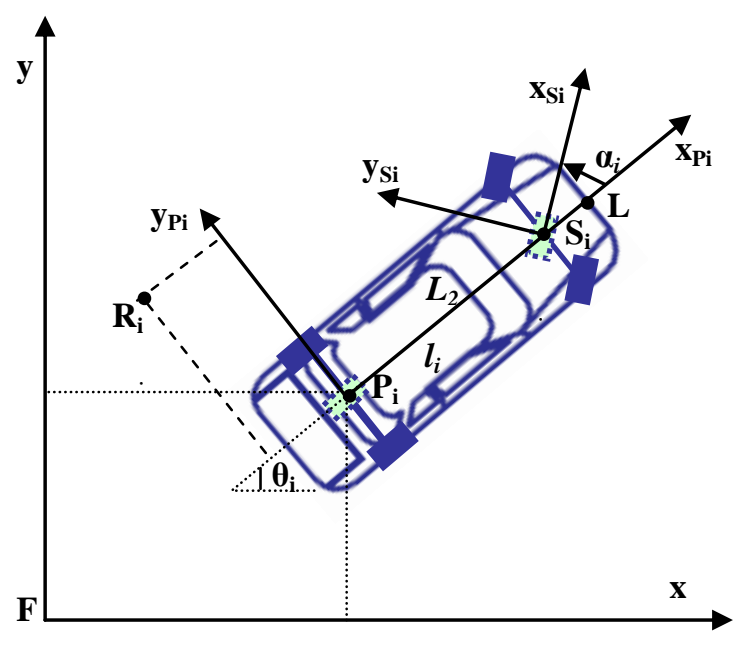




$$
{ }^{F} p_{R_{j}}={ }^{F} T_{P_{1}}{ }^{P_{1}} p_{R_{j}}
$$

Fig. 2. Coordinate frame assignment

where

The angle $\theta_{i},(i=1,2)$, is the orientation angle of the vehicle with respect to the frame Fxy. The front wheel steering angle $\alpha_{i},(i=1,2)$, is measured with respect to the vehicle body.

Since the vehicle is assumed to move on a planar surface, in what follows, we use $3 x 3$ rather than $4 x 4$ homogeneous transformation matrices ${ }^{A} T_{B}$ to transform the coordinates of a point $G$ from coordinate system $B$ denoted by ${ }^{B} p_{G}$ to its corresponding coordinates ${ }^{A} p_{G}$ in the coordinate frame $A$.

Using the above notations, the assignment of the coordinate frames results in the following transformation matrices between the coordinate systems

$$
{ }^{F} p_{L}={ }^{F} T_{P_{2}}{ }^{P_{2}} p_{L}
$$

$$
{ }^{P_{i}} T_{S_{i}}=\left[\begin{array}{ccc}
\cos \alpha_{i} & -\sin \alpha_{i} & l_{i} \\
\sin \alpha_{i} & \cos \alpha_{i} & 0 \\
0 & 0 & 1
\end{array}\right]
$$

(1)

Similarly, the homogeneous coordinates of the front reference point $L$ associated with the overtaking vehicle in frame $F x y$ are

where

$$
{ }^{P_{2}} p_{L}=\left[\begin{array}{lll}
L_{2} & 0 & 1
\end{array}\right]^{T} .
$$

\section{2) Nonholonomic constraints}

If the rotation of the wheels with respect to their proper axes is ignored, the vehicle configuration can be described by four generalized coordinates

$$
{ }^{F} T_{P_{i}}=\left[\begin{array}{ccc}
\cos \theta_{i} & -\sin \theta_{i} & F x_{P i} \\
\sin \theta_{i} & \cos \theta_{i} & F y_{P i} \\
0 & 0 & 1
\end{array}\right] .
$$

The transformation matrices (1) and (2) are applied to determine the position kinematics of the vehicle.

Using (1) and (2), the position of point $S_{i}$ in the inertial frame $F$ is

$$
\begin{aligned}
{ }^{F} p_{S_{i}} & ={ }^{F} T_{P_{i}}{ }^{P_{i}} T_{S_{i}}{ }^{S_{i}} p_{S_{i}} \\
& =\left[\begin{array}{ccc}
\cos \theta_{i} & -\sin \theta_{i} & { }^{F} x_{A_{i}} \\
\sin \theta_{i} & \cos \theta_{i} & F_{A_{i}} \\
0 & 0 & 1
\end{array}\right]\left[\begin{array}{ccc}
\cos \alpha_{i} & -\sin \alpha_{i} & l_{i} \\
\sin \alpha_{i} & \cos \alpha_{i} & 0 \\
0 & 0 & 1
\end{array}\right]\left[\begin{array}{l}
0 \\
0 \\
1
\end{array}\right] \\
& =\left[\begin{array}{c}
x_{P_{i}}+l_{i} \cos \theta_{i} \\
y_{P_{i}}+l_{i} \sin \theta_{i} \\
1
\end{array}\right] .
\end{aligned}
$$

$$
q_{i}=\left[{ }^{F} x_{P_{i}},{ }^{F} y_{P_{i}}, \theta_{i}, \alpha_{i}\right]^{T} \in \mathfrak{R}^{4} .
$$

Differentiating (3), the components of the velocity of point $S_{i}$ with respect to the inertial frame Fxy and expressed in Fxy are

$$
{ }^{F} \dot{p}_{S_{i}}=\left[\begin{array}{c}
{ }^{F} \dot{x}_{S_{i}} \\
{ }^{F} \dot{y}_{S_{i}} \\
0
\end{array}\right]=\left[\begin{array}{c}
{ }^{F} \dot{x}_{P_{i}}-l_{i} \dot{\theta}_{i} \sin \theta_{i} \\
F \dot{y}_{P_{i}}+l_{i} \dot{\theta}_{i} \cos \theta_{i} \\
0
\end{array}\right] .
$$

In order to derive the nonholonomic constraints of the front virtual wheel, the velocity of point $S_{i}$ relative to frame $F x y$ is expressed in frame $S_{i} x_{S i} y_{S i}$ as follows

Using (2), the homogeneous coordinates of the reference point $R_{j}$ associated with the overtaken vehicle in frame Fxy are 


$$
\begin{aligned}
{ }^{S_{i}} \dot{p}_{S_{i}} & =\left[\begin{array}{c}
{ }^{S_{i}} v_{S_{i} x} \\
{ }^{S_{i}} v_{S_{i} y} \\
0
\end{array}\right]=\left({ }^{F} T_{P_{i}}{ }^{A_{i}} T_{S_{i}}\right)^{-1} F \dot{p}_{S_{i}} \\
& =\left[\begin{array}{ccc}
\cos \left(\theta_{i}+\alpha_{i}\right) & \sin \left(\theta_{i}+\alpha_{i}\right) & * \\
-\sin \left(\theta_{i}+\alpha_{i}\right) & \cos \left(\theta_{i}+\alpha_{i}\right) & * \\
0 & 0 & 1
\end{array}\right]\left[\begin{array}{c}
F \dot{x}_{S_{i}} \\
F \dot{y}_{S_{i}} \\
0
\end{array}\right] .
\end{aligned}
$$

$$
C_{i}(q) \dot{q}_{i}=\mathrm{O}
$$

where $C_{i}\left(q_{i}\right)$ is a $2 \times 4$ full rank matrix of the form

$$
C_{i}(q)=\left[\begin{array}{cccc}
-\sin \theta_{i} & \cos \theta_{i} & 0 & 0 \\
-\sin \left(\theta_{i}+\alpha_{i}\right) & \cos \left(\theta_{i}+\alpha_{i}\right) & l_{i} \cos \alpha_{i} & 0
\end{array}\right]
$$

where the terms indicated by $(*)$ are irrelevant in the computation. Based on the assumption of rolling without lateral sliding, we have ${ }^{S_{i}} v_{S_{i} y}=0$, where ${ }^{S_{i}} v_{S_{i} y}$ is the component of the velocity of point $S_{i}$ along the $y$ axis of frame $S_{i} x_{S i} y_{S i}$. From the second line of equality (10) and by using expressions (9) for ${ }^{F} \dot{p}_{S_{i}}$, the nonholonomic constraint for the front virtual wheel can be written in the form

$$
0=-{ }^{F} \dot{x}_{P_{i}} \sin \left(\theta_{i}+\alpha_{i}\right)+{ }^{F} \dot{y}_{P_{i}} \cos \left(\theta_{i}+\alpha_{i}\right)+l_{i} \dot{\theta}_{i} \cos \alpha_{i} .
$$

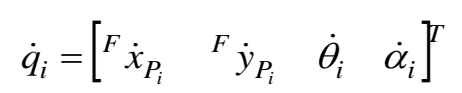

is the vector of generalized velocities. The constraint equation (14) can be converted into an affine driftless control system

$$
\dot{q}_{i}=D_{i}\left(q_{i}\right) \eta_{i}
$$

Likewise, using (2), the nonholonomic constraint imposed on the rear virtual wheel can be derived from the second line of the following expression

$$
\begin{aligned}
{ }^{P_{i}} \dot{p}_{P_{i}} & =\left[\begin{array}{c}
{ }^{P_{i}} v_{P_{i} x} \\
{ }^{P_{i}} v_{P_{i} y} \\
0
\end{array}\right]={ }^{F} T_{P_{i}}{ }^{-1 F} \dot{p}_{P_{i}} \\
& =\left[\begin{array}{ccc}
\cos \theta_{i} & \sin \theta_{i} & * \\
-\sin \theta_{i} & \cos \theta_{i} & * \\
0 & 0 & 1
\end{array}\right]\left[\begin{array}{c}
F \dot{x}_{P_{i}} \\
F \dot{y}_{P_{i}} \\
0
\end{array}\right] .
\end{aligned}
$$

where the columns of the $4 \times 2$ matrix $D_{i}\left(q_{i}\right)$

$$
D_{i}\left(q_{i}\right)=\left[\begin{array}{cc}
\cos \theta_{i} & 0 \\
\sin \theta_{i} & 0 \\
\frac{\tan \alpha_{i}}{l_{i}} & 0 \\
0 & 1
\end{array}\right]
$$

form a basis of the null space of $C_{i}\left(q_{i}\right)$. The control input $\eta_{i}=\left[{ }^{P_{i}} v_{P_{i} x}, \omega_{\alpha i}\right]^{T}$ is a $2 \mathrm{x} l$ vector of independent quasivelocities, which parameterizes the degree of freedom of the system, where ${ }^{P_{i}} v_{P_{i} x}$ is the velocity of point $P_{i}$, (the midpoint of the rear virtual wheel), and $\omega_{\alpha i}$ is the steering angular velocity of the front wheel. In this paper, the vehicle angular velocity (the front wheel steering angle $\alpha_{2}$, respectively) is considered as a control input instead of the steering angle velocity. From the third equation of (18), the front wheel steering angle can be expressed in terms of the vehicle angular velocity as follows

$$
\alpha_{i}=a \tan \left(l_{i} \frac{\dot{\theta}_{i}}{P_{i} v_{P_{i} x}}\right)
$$


Let us denote by

$$
F_{R_{R_{j}}}=\left[\begin{array}{lll}
F x_{R_{j}} & F y_{R_{j}} & \theta_{1}
\end{array}\right]^{T} \in \mathfrak{R}^{3}
$$

the posture of the overtaken vehicle in the inertial frame Fxy expressed in terms of the coordinates of reference point $R_{j}$. Differentiating (4) and using the first two equations of (18) for $i=1$, a kinematic model of the overtaken vehicle based on the $j^{\text {th }}$ the reference point $R_{j},(j=1,2,3)$, can be written in the form

$$
{ }^{F} \dot{z}_{R_{j}}=A_{1} \eta_{e 1}
$$

where

$$
A_{1}=\left[\begin{array}{ccc}
\cos \theta_{1} & 0 & -L_{t j} \sin \theta_{1}+L_{n j} \cos \theta_{1} \\
\sin \theta_{1} & 0 & L_{t j} \cos \theta_{1}-L_{n j} \sin \theta_{1} \\
0 & 0 & 1
\end{array}\right]
$$

$$
\eta_{e 1}=\left[\begin{array}{lll}
{ }^{P_{1}} v_{P_{1} x} & 0 & \omega_{1}
\end{array}\right]^{T}
$$

and $\omega_{1}:=\dot{\theta}_{1}$ is the angular velocity of the overtaken vehicle.

Similarly, let us denote by

$$
{ }^{F} z_{L}=\left[\begin{array}{llll}
F & & F & \\
x_{L} & & y_{L} & \theta_{2}
\end{array}\right]^{T} \in \mathfrak{R}^{3}
$$

the posture of the overtaking vehicle in the inertial frame Fxy expressed in terms of the coordinates of reference point L. Differentiating (6) and using the first two equations of (18) for $i=2$, a kinematic model of the overtaking vehicle based on the reference point $L$ is obtained as follows

$$
{ }^{F} \dot{z}_{L}=A_{2} \eta_{e 2}
$$

where

$$
A_{2}=\left[\begin{array}{ccc}
\cos \theta_{2} & 0 & L_{2} \sin \theta_{2} \\
\sin \theta_{2} & 0 & L_{2} \cos \theta_{2} \\
0 & 0 & 1
\end{array}\right]
$$

$$
\eta_{e 2}=\left[\begin{array}{lll}
{ }^{P_{2}} v_{P_{2} x} & 0 & \omega_{2}
\end{array}\right]^{T}
$$

and $\omega_{2}:=\dot{\theta}_{2}$ is the angular velocity of the overtaking vehicle.

\section{Relative Kinematics}

A schematic plan view of an overtaking maneuver involving two vehicles is shown in Fig. 3.

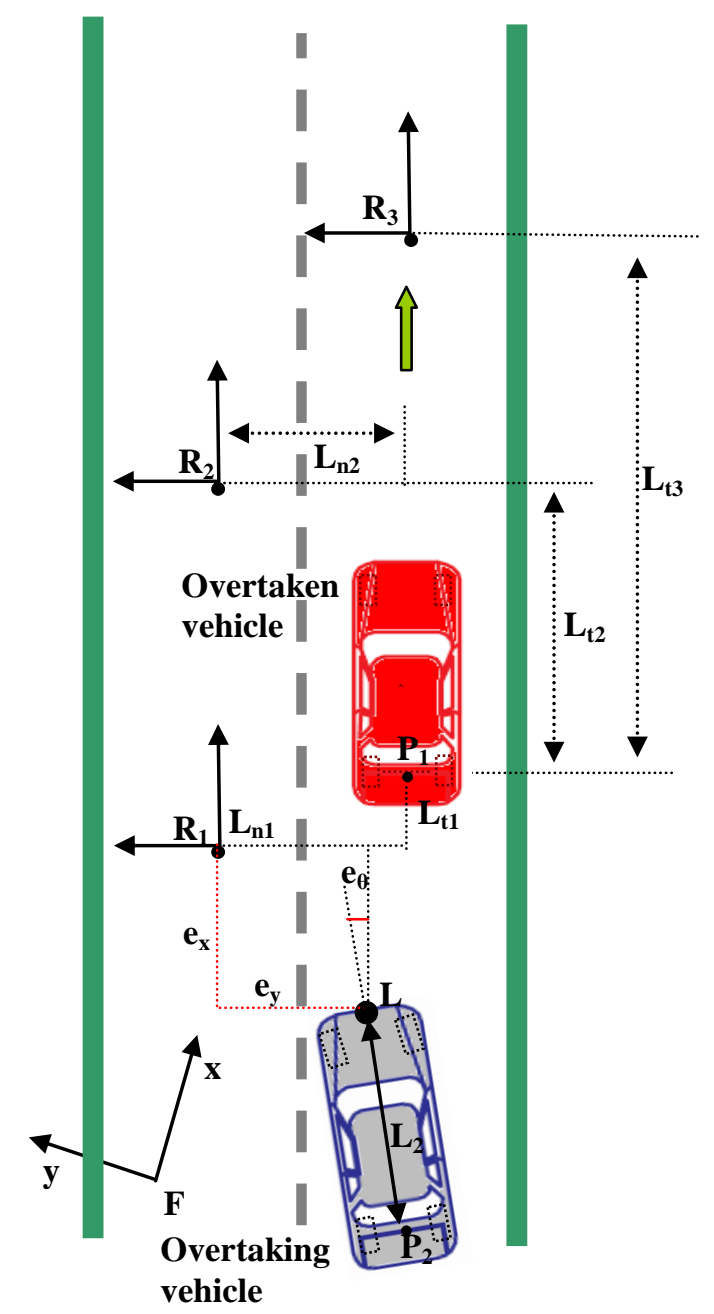

Fig. 3. Schematics of the overtaking maneuver

The coordinate frames $R_{j} x_{R j} y_{R j}(j=1,2,3)$ and $L x_{L} y_{L}$ are defined to describe the relative inter-vehicle kinematics during the first phase of the overtaking maneuver. For this purpose, we define a error posture, (the coordinates and orientation of the frame $L x_{L} y_{L}$ in the coordinate frame $\left.R_{j} x_{R j} y_{R j}\right)$, by the vector

$$
e_{j}=\left[\begin{array}{lll}
e_{x j} & e_{y j} & e_{\theta j}
\end{array}\right]^{T} \in \mathfrak{R}^{3}
$$


Using (20) and (24), the posture error vector can be expressed as follows [41]

$$
e_{j}=R\left({ }^{F} z_{L}-{ }^{F} z_{R_{j}}\right)
$$

where

$$
R=\left[\begin{array}{ccc}
\cos \theta_{1} & \sin \theta_{1} & 0 \\
-\sin \theta_{1} & \cos \theta_{1} & 0 \\
0 & 0 & 1
\end{array}\right]
$$

Differentiating (29) with respect to time and taking into account equations (21) and (25), after some work the intervehicle kinematics in error coordinates is obtained as follows

$$
\begin{aligned}
{\left[\begin{array}{c}
\dot{e}_{x j} \\
\dot{e}_{y j} \\
\dot{e}_{\theta j}
\end{array}\right] } & =\left[\begin{array}{ccc}
\cos e_{\theta j} & 0 & -L_{2} \sin e_{\theta j} \\
\sin e_{\theta j} & 0 & L_{2} \cos e_{\theta j} \\
0 & 0 & 1
\end{array}\right]\left[\begin{array}{c}
v_{2 x} \\
0 \\
\omega_{2}
\end{array}\right]-\left[\begin{array}{ccc}
1 & 0 & -L_{n j} \\
0 & 0 & -L_{t j} \\
0 & 0 & 1
\end{array}\right]\left[\begin{array}{c}
v_{1 x} \\
0 \\
\omega_{1}
\end{array}\right] \\
& +\omega_{1}\left[\begin{array}{ccc}
0 & 1 & 0 \\
-1 & 0 & 0 \\
0 & 0 & 0
\end{array}\right]\left[\begin{array}{c}
e_{x j} \\
e_{y j} \\
e_{\theta j}
\end{array}\right]
\end{aligned}
$$

where $v_{i x}:={ }^{P_{i}} v_{P_{i} x},(i=1,2)$, are the longitudinal components of the linear velocities of points $P_{1}$ and $P_{2}$, respectively associated with the overtaken and overtaking vehicle, respectively. We note that based on the assumption of pure rolling without lateral sliding of the wheels, the nonholonomic constraints imply that in (30) the components of the velocities of points $P_{1}$ and $P_{2}$ in direction of the rear vehicle axles are equal to zero, i.e., $v_{i y}:={ }^{P_{i}} v_{P_{i} y}=0,(i=1,2)$. In the following, we consider an overtaking maneuver during rectilinear motion of the overtaken vehicle. In this case, the angular velocity of the overtaking vehicle $\omega_{1}=0$.

Let us consider the following change of inputs in (30)

$$
\left[\begin{array}{l}
u_{1} \\
u_{2}
\end{array}\right]=\left[\begin{array}{cc}
\cos e_{\theta j} & -L_{2} \sin e_{\theta j} \\
\operatorname{sine}_{\theta j} & \mathrm{~L}_{2} \cos e_{\theta j}
\end{array}\right]\left[\begin{array}{c}
v_{2 x} \\
\omega_{2}
\end{array}\right]
$$

where one easy verify that the transformation matrix is nonsingular when $L_{2} \neq 0$. Using (31), in case of rectilinear motion of the overtaken vehicle, the inter-vehicle kinematic equations (4) can be written in the form

$$
\begin{aligned}
& \dot{e}_{x j}=u_{1}-v_{2 x} \\
& \dot{e}_{y j}=u_{2} \\
& \dot{e}_{\theta j}=-\frac{\sin e_{\theta j}}{L_{2}} u_{1}+\frac{\cos e_{\theta j}}{L_{2}} u_{2}
\end{aligned}
$$

\section{PROBLEM FormULATION}

In this paper, we consider autonomous overtaking maneuver without any information obtained from road infrastructure or communicated from the overtaken vehicle. The only information that the overtaking (automatic) vehicle can use for feedback control is the current relative position and orientation with respect to the overtaken vehicle given by (28), which are obtained from onboard sensors.

For brevity, in the exposition which follows, we will derive in details the trajectory planning procedure with respect to the first phase of the overtaking maneuver (lane change maneuver). Similar relationships can be also derived for Phase 2 and Phase 3. The index 1 will be also omitted and we will denote the posture error $e_{1}=\left[\begin{array}{lll}e_{x 1} & e_{y 1} & e_{\theta 1}\end{array}\right]^{T}$ by $e=\left[\begin{array}{lll}e_{x} & e_{y} & e_{\theta}\end{array}\right]^{T}$

\section{A. Trajectory Planning}

We consider in details the problem of generating smooth trajectories for point to point motion in terms of the posture errors $\left(e_{x}, e_{y}\right)$ with respect to the moving reference frame $R_{l} x_{R l} y_{R l}$, which is rigidly linked to the overtaken vehicle. Assuming rectilinear motion at constant velocity of the overtaken vehicle, we are interested in overtaking scenario for the first phase, when the overtaking vehicle starts the maneuver from an arbitrary initial position and orientation behind the overtaken vehicle with given (arbitrary) velocity. In the end of the first phase, the two vehicles have to be parallel and in the same time, the desired position of the overtaking vehicle behind the overtaken vehicle has to be shifted at distances $L_{t 1}$ and $L_{n 1}$ in longitudinal and lateral direction, respectively, (Fig. 3). These distances are determined from considerations of safety driving and sensor requirements (in particular, $L_{t 1}$ may be equal to zero). In addition, the relative intervehicle velocity in longitudinal direction must be equal to a prescribed non-zero value. For this end, we suppose that at time $t_{0}$, the state variables satisfy

$$
\begin{aligned}
& e_{x}^{d}\left(t_{0}\right)=e_{x 0} \\
& \dot{e}_{x}^{d}\left(t_{0}\right)=\dot{e}_{x 0} \\
& e_{y}^{d}\left(t_{0}\right)=e_{y 0} \\
& \dot{e}_{y}^{d}\left(t_{0}\right)=\dot{e}_{y 0}
\end{aligned}
$$

At time $t_{f}$, we wish to attain the values 


$$
\begin{gathered}
e_{x}^{d}\left(t_{f}\right)=0 \\
\dot{e}_{x}^{d}\left(t_{f}\right)=\Delta v_{R x}\left(t_{f}\right)
\end{gathered}
$$

$$
\begin{aligned}
& e_{y}^{d}\left(t_{f}\right)=0 \\
& \dot{e}_{y}^{d}\left(t_{f}\right)=0
\end{aligned}
$$

where $\Delta v_{R x}\left(t_{f}\right)$ is the prescribed relative non-zero inter-vehicle velocity in $x_{R I}$-direction at $t_{f}$, i.e., the overtaking vehicle has to start the second phase of the overtaking maneuver at prescribed velocity which is higher compared to the velocity of the overtaken vehicle. A specific feature of the trajectory planning procedure is that the initial values of the program errors $\left(e_{x}^{d}\left(t_{0}\right), e_{y}^{d}\left(t_{0}\right)\right)$ are equal of the current values $\left(e_{x}\left(t_{0}\right), e_{y}\left(t_{0}\right)\right)$ at $t=t_{0}$, and in such way, smooth transition between the adjacent phases is assured. The time $t_{f}$, is determined from considerations of vehicle's power and acceleration capabilities. However, from a practical point of view, the following conditions have to be satisfied: $a_{x} \leq e_{x}\left(t_{0}\right) \leq b_{x}, \quad a_{y} \leq e_{y}\left(t_{0}\right) \leq b_{y}$, where $a_{x}, b_{x}, a_{y}$, and $b_{y}$ are determined from considerations of safety driving, sensor requirements, and vehicle's power and acceleration capabilities. In this paper, we consider cubic polynomials for the desired trajectories of the form

$$
\begin{aligned}
& e_{x}^{d}(t)=a_{0 x}+a_{1 x}\left(t-t_{0}\right)+a_{2 x}\left(t-t_{0}\right)^{2}+a_{3 x}\left(t-t_{0}\right)^{3} \\
& e_{y}^{d}(t)=a_{0 y}+a_{1 y}\left(t-t_{0}\right)+a_{2 y}\left(t-t_{0}\right)^{2}+a_{3 y}\left(t-t_{0}\right)^{3}
\end{aligned} .
$$

Differentiating (35) with respect to time, we obtain quadratic polynomials with respect to the derivatives $\dot{e}_{x}^{d}(t)$ and $\dot{e}_{y}^{d}(t)$ :

$$
\begin{aligned}
& \dot{e}_{x}^{d}(t)=a_{1 x}+2 a_{2 x}\left(t-t_{0}\right)+3 a_{3 x}\left(t-t_{0}\right)^{2} \\
& \dot{e}_{y}^{d}(t)=a_{1 y}+2 a_{2 y}\left(t-t_{0}\right)+3 a_{3 y}\left(t-t_{0}\right)^{2}
\end{aligned}
$$

Using (33), (34), (35) and (36), we obtain eight equations for the eight unknown coefficients $a_{i x}$ and $a_{i y}$, $(i$ $=1,2,3,4)$. Similar expressions for the desired trajectories can be assigned for Phase 2 and Phase 3.

\section{B. Problem Statement}

We assume that we are able to measure the posture coordinates $e=\left[\begin{array}{lll}e_{x} & e_{y} & e_{\theta}\end{array}\right]^{T} \in \mathfrak{R}^{3}$ defined in (28), but the linear velocity $v_{l x}$ of the overtaken vehicle is unknown constant parameter.

Given the inter-vehicle kinematics in error coordinates (32), the control objective for Phase 1 is to asymptotically regulate to zero the coordinates $\left(e_{x}, e_{y}\right)$ of the reference point $L$ of the overtaking vehicle with respect to the coordinate frame $R_{l}$ (attached to the overtaken vehicle) in accordance with the desired trajectories (35) with respect to the error coordinates $\left(e_{x}, e_{y}\right)$ with initial and final conditions given by (33)-(34). Similar objectives can be formulated for Phase 2 and Phase 3, where the virtual reference points associated with the overtaken vehicle are $R_{2}$ and $R_{3}$, respectively.

\section{ADAPTIVE CONTROL DESIGN}

In this paper, we consider the problem of controlling the motion of the overtaking vehicle during the overtaking maneuver, when the overtaken vehicle moves straight with constant velocity. Consider again in details the Phase 1 of the overtaking maneuver. We make the following change of coordinates

$$
\begin{aligned}
& x_{e}=e_{x}-e_{x}^{d} \\
& y_{e}=e_{y}-e_{y}^{d}
\end{aligned}
$$

where the posture error coordinates $\left(e_{x}, e_{y}\right)$ are defined by (29), and the desired trajectories $\left(e_{x}^{d}(t), e_{y}^{d}(t)\right)$ are given by (35).

The model (32) describing inter-vehicle kinematics in error coordinates is redefined in terms of the new coordinates (37) as follows

$$
\begin{aligned}
& \dot{x}_{e}=u_{1}-v_{1 x}-\dot{e}_{x}^{d} \\
& \dot{y}_{e}=u_{2}-\dot{e}_{y}^{d} \\
& \dot{e}_{\theta}=-\frac{\sin e_{\theta}}{L_{2}} u_{1}+\frac{\cos e_{\theta}}{L_{2}} u_{2}
\end{aligned}
$$

The adaptive control design is based on a reduced-order system composed of the first two equations of (38) rewritten below for clarity of exposition

$$
\begin{aligned}
& \dot{x}_{e}=u_{1}-v_{1 x}-\dot{e}_{x}^{d} \\
& \dot{y}_{e}=u_{2}-\dot{e}_{y}^{d}
\end{aligned}
$$

Consider the system (39) and assume that the velocity of the overtaken vehicle $v_{1 x}=$ cte $>0$ is unknown constant parameter. The control problem consists in finding an adaptive feedback control law for the system (39) with inputs $\left(u_{1}, u_{2}\right)$ such that

$$
\lim _{t \rightarrow \infty}\left(x_{e}(t)\right)=0 \quad \text { and } \quad \lim _{t \rightarrow \infty}\left(x_{e}(t)\right)=0 .
$$

Consider the control

$$
\begin{aligned}
& u_{1}=\hat{v}_{1 x}+\dot{e}_{x}^{d}-k_{x} x_{e} \\
& u_{2}=\dot{e}_{y}^{d}-k_{y} y_{e}
\end{aligned}
$$


where $k_{x}$ and $k_{y}$ are positive gains, and $\hat{v}_{1 x}$ is the estimate of the overtaken vehicle velocity. We chose the following Lyapunov function candidate

$$
V=\frac{1}{2} x_{e}^{2}+\frac{1}{2} y_{e}^{2}+\frac{1}{2 \gamma_{v}} \tilde{v}_{1 x}^{2}
$$

where

$$
\tilde{v}_{1 x}=\hat{v}_{1 x}-v_{1 x}
$$

is the parameter error, and $\gamma_{v}=$ cte $>0$ is the adaptation gain. Using (39), (41) and (43), the derivative of $V$ is obtained in the form

$$
\dot{V}=-k_{x} \dot{x}_{e}^{2}-k_{y} \dot{y}_{e}^{2}+\tilde{v}_{1 x}\left(x_{e}+\frac{1}{\gamma_{v}} \dot{\hat{v}}_{1 x}\right) \text {. }
$$

Choosing the update law as

$$
\dot{\hat{v}}_{1 x}=-\gamma_{v} x_{e}
$$

we get for the derivative of $V$

$$
\dot{V}=-k_{x} \dot{x}_{e}^{2}-k_{y} \dot{y}_{e}^{2} \leq 0 \text {. }
$$

The resulting closed-loop adaptive system becomes

$$
\begin{aligned}
& \dot{x}_{e}=-k_{x} x_{e}+\tilde{v}_{1 x} \\
& \dot{y}_{e}=-k_{y} y_{e} \\
& \dot{\tilde{v}}_{1 x}=-\gamma_{v} x_{e}
\end{aligned} .
$$

Proposition 1: Assume that the linear velocity of the overtaken vehicle is bounded unknown constant parameter $v_{1 x}=c t e>0$ and also that $L_{2} \neq 0$. If the control law given by (41) is applied to (39), where the velocity estimate $\hat{v}_{1 x}$ is obtained from the parameter update law (45), the origin $o_{Z}=\left[x_{e}, y_{e}, \tilde{v}_{1 x}\right]^{T}=0$ of the closed-loop system (47) is asymptotically stable.

Proof. The system (47) has an equilibrium point at the origin. The function (42) is continuously differentiable and positive definite, and its derivative (46) along the trajectories of the system is negative semi-definite. From (46), it follows that (42) is non-increasing, $(V(t) \leq V(0))$, and this in turn implies that $x_{e}(t), y_{e}(t)$ and $\tilde{v}_{1 x}(t)$ are uniformly bounded with respect to the initial conditions.

To characterize the set $S=\left\{o_{Z} \in \mathfrak{R}^{3} \mid \dot{V}(z)=0\right\}$, note that

$$
\dot{V}(z)=0 \Rightarrow x_{e}=0 \quad \text { and } \quad y_{e}=0 .
$$

Hence $S=\left\{o_{Z} \in \mathfrak{R}^{3} \mid x_{e}=0 ; \quad y_{e}=0\right\} . \quad$ To prove asymptotic stability of the equilibrium point $o_{Z}=0$, we use the LaSalle invariance principle [42]. Suppose that $o_{Z}(t)$ is a trajectory that belongs identically to $S$. From (46), we have

$$
\begin{aligned}
& x_{e}(t) \equiv 0 \Rightarrow \dot{x}_{e} \equiv 0 \Rightarrow \tilde{v}_{1 x} \equiv 0 \\
& y_{e}(t) \equiv 0 \Rightarrow \dot{y}_{e} \equiv 0 \\
& x_{e}(t) \equiv 0 \Rightarrow \dot{\tilde{v}}_{1 x} \equiv 0
\end{aligned}
$$

Therefore, the only solution that can stay in $S$ is the trivial solution $o_{z}(t)=0$ and the origin is asymptotically stable.

Since the dynamics of $e_{\theta}$ was not taken into account in the feedback control design, the next step in the stability analysis is to establish that $e_{\theta}$ is bounded in the interval [ $t_{0}$, $t_{f}$. From (38), using the control (41), the third equation for $e_{\theta}$ takes the form of a perturbed system

$$
\dot{e}_{\theta}=f\left(e_{\theta}\right)+g\left(t, e_{\theta}\right)
$$

where the nominal system is given by

$$
\dot{e}_{\theta}=f\left(e_{\theta}\right)=-\frac{v_{1 x}}{L_{2}} \sin e_{\theta},
$$

and the perturbation term is obtained in the form

$$
\begin{aligned}
g\left(t, e_{\theta}\right) & =\frac{1}{L_{2}}\left[-\left(\tilde{v}_{1 x}-k_{x} x_{e}\right) \sin e_{\theta}-k_{y} y_{e} \cos e_{\theta}\right. \\
& \left.-\dot{e}_{x}^{d} \sin e_{\theta}+\dot{e}_{y}^{d} \cos e_{\theta}\right]
\end{aligned}
$$

Since $x_{e}(t), y_{e}(t)$ and $\tilde{v}_{1 x}(t)$ are uniformly bounded, $\left(\sin \left(e_{\theta}\right)\right.$ and $\cos \left(e_{\theta}\right)$ are bounded functions), and the polynomial functions (36) are bounded within the interval $\left[t_{0}, t_{f}\right]$, it follows that

$$
\left\|g\left(t, e_{\theta}\right)\right\| \leq \lambda
$$

in the domain of interest, ( $\lambda$ is a positive constant).

The point $e_{\theta}=0$ is an exponentially stable equilibrium point for the nominal system (51), which can be easy proved by using, for example, the Lyapunov function $W=1-\cos e_{\theta}$. Therefore, $e_{\theta}$ is bounded and $e_{\theta}(t) \rightarrow 0$ as $t \rightarrow \infty$. If $e_{\theta n}(t)$ and $e_{\theta p}(t)$ are the solutions of the nominal 
and perturbed systems (51) and (50), respectively, using the Gronwall-Bellman inequality [42], we have on the compact time interval $\left[t_{0}, t_{f}\right]$

$$
\begin{gathered}
\left\|e_{\theta n}(t)-e_{\theta p}(t)\right\| \leq \\
+e_{\theta n}\left(t_{0}\right)-e_{\theta 0}(t) \| \exp \left[l\left(t-t_{0}\right)\right] \\
+\frac{\lambda}{l}\left\{\exp \left[l\left(t-t_{0}\right)\right]-1\right\}
\end{gathered}
$$

where $l$ is a Lipschitz constant for the nominal system (51). Hence, $e_{\theta p}(t)$ is bounded on the time interval $\left[t_{0}, t_{f}\right]$.

The designed control law (41)-(45) constitutes the upper level controller of the overtaking vehicle and provides reference signals to the lower level controller for the steering and velocity dynamics, which have to be tracked. As mentioned above, at the end of the first time period, the guidance program switches automatically from Phase 1 to Phase 2. The same approach can be used to derive feedback controllers for the overtaking vehicle for Phase 2 and Phase 3.

\section{Simulation Results}

To illustrate the effectiveness of the proposed controller, several simulations are carried out in order to evaluate the inter-vehicle behavior and tracking accuracy during the three-phase overtaking maneuver. In the simulation using MATLAB, a planar bicycle 2DOFs vehicle model is used. The speed of the overtaken vehicle during the three phases of the maneuver is constant and set to be $v_{l x}=4 \mathrm{~m} / \mathrm{s}$. The duration of every phase of the maneuver is chosen to be $t_{i n t}=5 \mathrm{~s}$. The coordinates of the reference frames

TABLE I

COORDINATES OF THE REFERENCE FRAMES $\mathrm{R}_{\mathrm{I}} \mathrm{X}_{\mathrm{RI}} \mathrm{Y}_{\mathrm{RI}},(\mathrm{I}=1,2,3)$, IN THE OVERTAKEN VEHICLE FRAME $\mathrm{P}_{1} \mathrm{X}_{\mathrm{P} 1} \mathrm{YP}_{\mathrm{P}}$

\begin{tabular}{lll}
\hline \hline \multirow{2}{*}{ Phase } & Reference Frame & \multicolumn{1}{c}{ Coordinates } \\
\hline 1 & $\mathrm{R}_{1} \mathrm{x}_{\mathrm{R} 1} \mathrm{y}_{\mathrm{R} 1}$ & $\mathrm{~L}_{\mathrm{t} 1}=-1 \mathrm{~m} ; \mathrm{L}_{\mathrm{n} 1}=3 \mathrm{~m}$ \\
2 & $\mathrm{R}_{2} \mathrm{x}_{\mathrm{R} 2} \mathrm{y}_{\mathrm{R} 2}$ & $\mathrm{~L}_{\mathrm{t} 2}=8 \mathrm{~m} ; \quad \mathrm{L}_{\mathrm{n} 2}=3 \mathrm{~m}$ \\
3 & $\mathrm{R}_{3} \mathrm{x}_{\mathrm{R} 3} \mathrm{y}_{\mathrm{R} 3}$ & $\mathrm{~L}_{\mathrm{t} 3}=12 \mathrm{~m} ; \mathrm{L}_{\mathrm{n} 3}=0 \mathrm{~m}$ \\
\hline \hline
\end{tabular}

$R_{j} x_{R j} y_{R j},(j=1,2,3)$ with respect to the coordinate frame $P_{1} x_{P 1} y_{P 1}$ attached to the mid-point of the rear vehicle axle of the overtaken vehicle for every phase of the three-phase overtaking maneuver is provided in Table I.

The longitudinal vehicle base of the overtaking vehicle was chosen to be $l_{2}=2 \mathrm{~m}$. The initial position and orientation of the overtaking vehicle in the inertial frame Fxy are ${ }^{F} x_{P 2}(0)=0 ;{ }^{F} y_{P 2}(0)=0 ; \theta_{2}(0)=0$. The initial position and orientation of the overtaken vehicle in Fxy are ${ }^{F} x_{P I}(8)=0 ;{ }^{F} y_{P I}(0)=0 ; \theta_{l}(0)=0$. The desired intervehicle distance in the beginning of the maneuver is set to be $6 m$.

In the first simulation, from Fig. 4, we can see the planar path drown by the vehicle guide points $P_{2}$ and $P_{1}$ of the overtaking and overtaken vehicle, respectively.

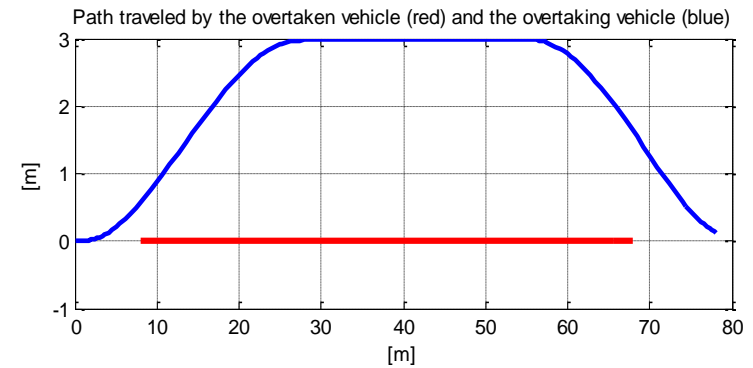

Fig. 4. Planar path drown by the vehicle guide points (the mid-points of the rear vehicle axles): point $P_{2}$ (blue line) and point point $P_{1}$ (red line).

From Fig. 5, the evolution in time of the error coordinates $x_{e}(t), y_{e}(\mathrm{t})$ is shown.

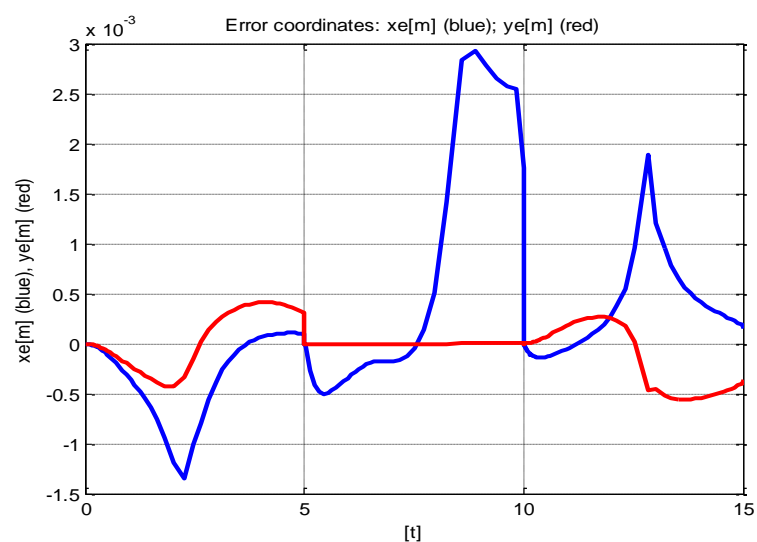

Fig. 5. Time history of the error coordinates $x_{e}(t)$ and $y_{e}(\mathrm{t})$.

The evolution in time of the error coordinate $e_{\theta}(t)$ is presented in Fig. 6.

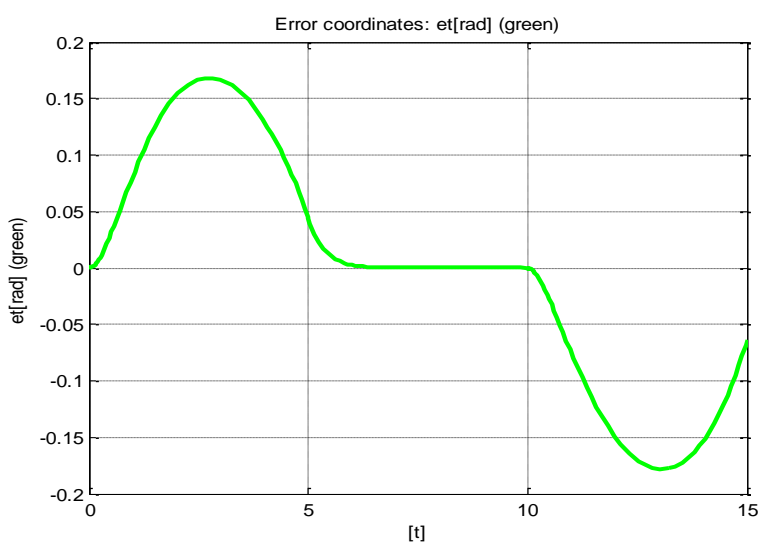

Fig. 6. Time history of the error coordinate $e_{\theta}(t)$.

As seen from Fig. 6, the relative orientation $e_{\theta}(t)$ between the two vehicles remains bound during the three phases of the overtaking maneuver, which is consistent with the theoretical results in Section IV.

The evolution in time of the reference trajectory $\left(e_{x}^{d}(t), \quad e_{y}^{d}(t)\right)$ is given in Fig.7. 


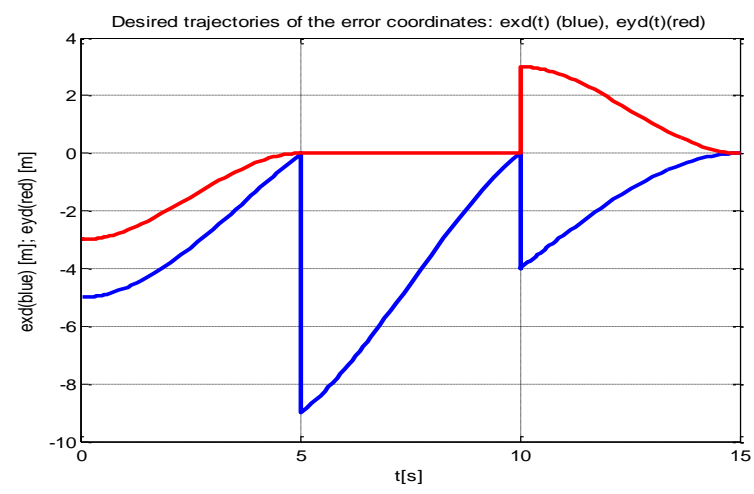

Fig. 7. Time history of the inter-vehicle distance $P_{l} L$

The evolution in time of the front-wheel steering angle $\alpha_{2}$ of the overtaking vehicle is given in Fig.8.

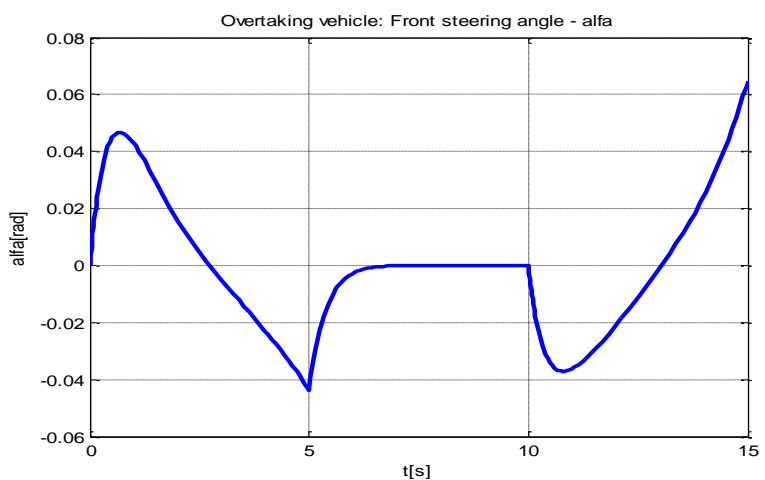

Fig. 8. Time history of the front-wheel steering angle of the overtaking vehicle

The evolution in time of the inter-vehicle distance $P_{l} L$ is given in Fig.9.

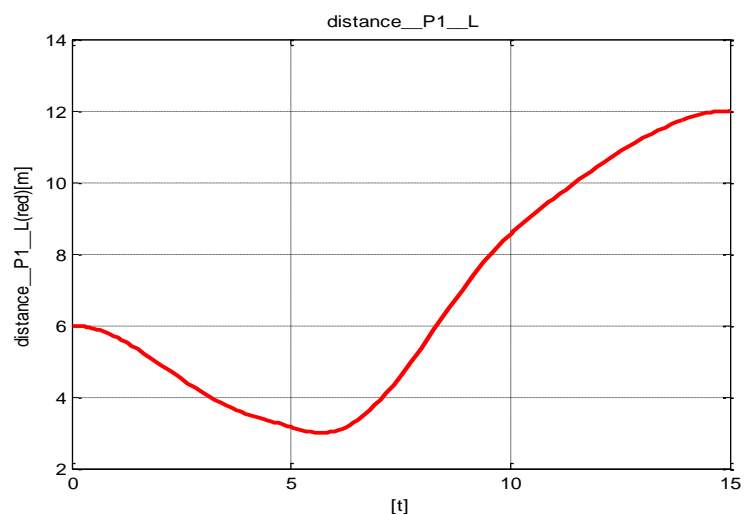

Fig. 9. Time history of the inter-vehicle distance $P_{l} L$

The results of the simulation confirm the validity of the proposed controller.

\section{CONCLUSION}

In this paper, a nonlinear adaptive controller for twovehicle automated overtaking maneuvers has been presented. We consider the problem of a three-phase overtaking without the use of information obtained from road infrastructure or inter-vehicle communication. Applying standard robotic nomenclature for translational and rotational displacements and velocities, a general kinematic model of the vehicles during the overtaking maneuver and the relative inter-vehicle kinematics has been developed. Reference trajectories for every phase using $3^{\text {rd }}$ order polynomial interpolation method have been generated in real-time and consecutively tracked. The developed feedback controller requires information only for the current relative inter-vehicle position and orientation. The unknown velocity of the overtaken vehicle has been estimated using adaptive update law. Simulation results illustrate the performance of the proposed controller. An autonomous overtaking control system may constitute a backup system for the infrastructure-based control system when the letter is malfunctioning or due to the failure the inter-vehicle communication.

\section{REFERENCES}

[1] J. Couplan, "Le systeme de transport urbain ARAMIS, in Proc. $1^{\text {st }}$ Int. Conf. Transp. Research," Bruges, Belgium, 1973, pp. $351-354$

[2] P. Daviet and M. Parent, "Platooning technique for empty vehicles distribution in the PRAXITELE," in Proc. $4^{\text {th }}$ IEEE Mediter. Symp. New Direct. Contr. Automation, Kreete, Greece, 1996, pp. 101-106.

[3] S. Shladover, "The GM-PATH Platoon Scenario," Intellimotion, Vol. 6, No 3, pp. 2-3 1997.

[4] Available at: http://www.autoroadvehicles.com/imtsexpo2005.html

[5] T. Fraichard, "CyberCar: L'alternative a la voiture particuliere, Navigation," 53(209), pp. 53-75 2009.

[6] Available at: http://www.trg.soton.ac.uk/stardust/index.htm

[7] A. Alessandrini and F. Filipi, "Ex-ante evaluation of nine cybernetics transport systems," in Proc. $7^{\text {th }}$ Int. Conf. Intel. Transportation Systems, 2004, pp. 994-999.

[8] CityMobil, European Commission, DG Research, $6^{\text {th }}$ Framework Programme, Thematic PriorityContract 1.6, No 031315.

[9] Available at: http://www.haveit-eu.org/.

[10] H. M. Lim and J. Karl Hedrick, "Lateral and longitudinal vehicle control coupling for automated vehicle operation," in Proc. Amer. Contr. Conference, San Diego, USA, 1999, pp. 3676-3680

[11] L. Li and F. Wang, Advanced motion control and sensing for intelligent vehicles, Springer, 2007.

[12] H. Peng and M. Tomizuka, "Lateral control of front-wheelsteering rubber-tire vehicles," UCB-ITS-PRR-90-5, 1990.

[13] R. Rajamani, Vehicle dynamics and control, Second edition, Springer, 2012.

[14] J. Balla, "Dynamics of mounted automatic cannon on track vehicle," Int. J. Math. Models and Methods in Appl. Sciences," Issue 3, Volume 5, pp. 423-432, 2011.

[15] P. Petrov and M. Parent, "Dynamic modeling and adaptive motion Control of a two-wheeled self-balancing vehicle for personal transport," in Proc. $13^{\text {th }}$ Int. IEEE Annual Conf. Intell. Transp. Systems, Madeira, Portugal, 2010 pp.1113115

[16] S. Wu, H. Chiang, J. Perng, T. Lee, and C. Chen, "The Automated Lane-keeping Design for an Intelligent Vehicle," in Proc. IEEE Int. Veh. Symposium, 2005, pp. 508-513.

[17] M. Oya and Q. Wang, "Adaptive lane keeping controller for four-wheel-steering vehicles," in Proc. IEEE Int. Conf. Contr. and Automation, Guangzhou, China, 2007, pp. 1942-1947.

[18] S. Mammar, "Two-degree-of-freedom $\mathrm{H}_{\infty}$ optimization and scheduling for robust vehicle lateral control," Vehicle System Dynamics, vol. 34, pp. 401-422, 2000. 
[19] A. Ferrara and C. Vecchio, "Cruise control with collision avoidance for cars via sliding modes," in Proc. IEEE Int. Conf. Contr. Applications, Munich, Germany. 2006, pp. 2808-2813.

[20] Y. Yoshida, Q. Wang, M. Oya, and K. Okumura, "Adaptive longitudinal velocity and lane keeping control of fourwheel-steering vehicles," in Proc. SICE Annual Conference, Kagawa University, Japan, 2007, pp. 1305-1310.

[21] H. Pham, M. Tomizuka, and J. Karl Hedrick, "Integrated Maneuvering Control for Automated Highway Systems Based on a Magnetic Reference/Sensing system," California PATH Research Report, UCB-ITS-PRR-97-28, 1997.

[22] Available

http://corporate.ford.com/innovation/innovationfeatures/innovation-detail/ford-new-lane-technology.

[23] T. Hessburg and M. Tomizuka, "Fuzzy Logic Control For Lane Change Maneuvers In Lateral Vehicle Guidance," UCB-ITS-PWP-95-13, 1995.

[24] M. Koopman, "The development of a path planning strategy for obstacle avoidance and crash impact minimization for an automatic guided vehicle," TNO report 03.OR.AC.034.1/MRK, 2003.

[25] W. Chee and M. Tomizuka, "Vehicle lane change maneuver in automated highway systems," California PATH Research Report, UCB-ITS-PRR-94-92, 1994.

[26] O. Mokhiamar and M. Abe, "Active wheel steering and yaw moment control combination to maximize stability as well as vehicle responsiveness during quick lane change for active vehicle handling safety," in Proc. Instn. Mech. Engrs, vol 216, Part D: J. Automobile Engineering, 2002, pp. 115124.

[27] A. Piazzi and C. Bianco, "Quantic G2-splines for trajectory planning of autonomous vehicles," in Proc. IEEE Int. Veh. Symposium, 2000, pp. 198-203.

[28] I. Papadimitriou, M. Tomizuka, Fast lane changing computations using polynomials, in Proc. Amer. Contr. Conference, 2003, pp. 48-53.

[29] Resende and F. Nashashibi, "Real-time dynamic trajectory planning for highly automated driving in highways," in IEEE Conf. Intel. Transp. Systems, 2010, pp. 653-658.

[30] C. Laugier, "Towards autonomous vehicles for future intelligent transportation systems," available at: http:// citeseerx.ist.psu.edu/viewdoc/summary?doi=10.1.1.52.4080

[31] W. Chee and M. Tomizuka, "Unified lateral motion Control of vehicles for lane change maneuvers in automated highway systems," California PATH Research Report UCBITS-PRR-97-29, 1997.

[32] C. Hatipoglu, K. Redmill, and U. Ozguner, "Steering and lane change: A working system," in Proc. Intel. Trans. Systems, Boston, USA, 1997, pp. 272-277.

[33] C. Hatipoglu, Ü. Özgüner, and K. Redmill, "Automated Lane Change Controller Design," IEEE Trans. Intel. Trans. Systems, Vol. 4, No. 1, pp. 13-22, 2003.

[34] P. Petrov and F. Nashashibi, "Planning and Nonlinear Adaptive Control for an AutomatedOvertaking Maneuver," in Proc. $14^{\text {th }}$ Int. IEEE Conf. Intel. Trans. Systems, Washington, USA, 2011, pp. 662-667.

[35] T. Shamir, Overtaking a slower-moving vehicle by an autonomous vehicle, in Proc. EIS2004, 2004.

[36] U. Ghumman, F. Kunwar, and B. Benhabib, "Guidancebased on-line motion planning for autonomous highway overtaking," Int. J. Smart Sensing and Intel. Systems, Vol. 1, No. 2, pp. 549-571, 2008.

[37] J. Naranjo, C. Gonzales, R. Garcia, and T. Pedro, "Lanechange fuzzy control in autonomous vehicles for the overtaking maneuver," IEEE Trans. Intel. Transp. Systems, vol. 9, no. 3, pp. 438-450, September, 2008.
[38] F. Wang, M. Yang, and R. Yang, "Conflict-probabilityestimation-based overtaking for intelligent vehicles," IEEE Trans. Intel. Trans. Systems, Vol. 10, No. 2, pp. 366-370, 2009.

[39] J. Baber, J. Kolodko, T. Noel, M. Parent, and L. Vlacic, "Cooperative autonomous driving: Intelligent vehicles sharing city roads," IEEE Robotics \& Automation Magazine, pp. 44-49, March, 2005.

[40] M. Spong and M. Vidyasagar, Robot dynamics and control, John Wiley\&Sons, 1988.

[41] P. Petrov, "Robust trajectory tracking algorithms for a wheeled mobile robot," in Proc. IEEE Ind. Electronics. Cont. and Instrumentation, 1991, pp. 1071-1074.

[42] H. Khalil, Nonlinear Systems. Second edition, PrenticeHall, Inc., 1996. 